\title{
Long term results of follow-up after HPV self-sampling with devices Qvintip and HerSwab in women non-attending cervical screening programme
}

\author{
Teodora Bokan', Urska Ivanus²,3, Tine Jerman², Iztok Takac,5, Darja Arko ${ }^{4,5}$ \\ ${ }^{1}$ Mistelbach-Gänserndorf Regional Hospital, Mistelbach Austria \\ ${ }^{2}$ Institute of Oncology Ljubljana, Ljubljana, Slovenia \\ ${ }^{3}$ Faculty of Medicine, University of Ljubljana, Ljubljana Slovenia \\ ${ }^{4}$ University Medical Centre Maribor, Maribor, Slovenia \\ ${ }^{5}$ Faculty of Medicine, University of Maribor, Maribor, Slovenia
}

Radiol Oncol 2021; 55(2): 187-195.

Received 1 October 2020

Accepted 9 December 2020

Correspondence to: Assoc. prof. Darja Arko, M.D., Ph.D., Division of Gynaecology and Perinatology, University Medical Centre Maribor, Ljubljanska 5, 2000 Maribor. E-mail: darja.arko@ukc-mb.si

Disclosure: No potential conflicts of interest were disclosed.

Background. We are presenting the results of the Slovenian human papillomaviruses (HPV) self-sampling pilot study in colposcopy population of National Cervical Cancer Screening Programme ZORA for the first time. One-year and four-year follow-up results are presented for two different self-sampling devices.

Participants and methods. A total of 209 women were enrolled in the study at colposcopy clinic. Prior to the gynaecological examination, all women performed self-collected vaginal swab at the clinic; 111 using Qvintip and 98 using HerSwab self-sampling device. After self-sampling, two cervical smears were taken by a clinician; first for conventional cytology and second for HPV test. After that, all women underwent colposcopy and a cervical biopsy if needed. We compared sensitivity, specificity, and predictive values of cytology (at the cut-off atypical squamous cells of undetermined significance or more [ASC-US+]) and HPV test (on self- and clinician-taken samples) for the detection of cervical intraepithelial neoplasia grade 2 or more $(\mathrm{CIN} 2+)$ after one and four years of follow-up. Hybrid Capture 2 (HC2) assay was used for all HPV testing.

Results. The mean age of 209 women was 37.6 years and HPV positivity rate $67.0 \%$ (140/209), 36.9 years and $70.3 \%$ (78/111) in the Qvintip group and 38.4 years and 63.3\% (62/98) in the HerSwab group, respectively. Overall, percent agreement between self and clinician-taken samples was $81.8 \%$ (kappa 0.534) in the Qvintip and 77.1\% (kappa 0.456) in the HerSwab group. In the Qvintip group, the longitudinal sensitivity, specificity, positive and negative predictive values were $71.8 \%, 75.0 \%, 83.6 \%, 60.0 \%$ for cytology; $83.1 \%, 51.3 \%, 75.6 \%$ and $62.5 \%$ for HPV test of self-taken samples and $94.4 \%, 57.5 \%, 79.8 \%$ and $85.2 \%$ for HPV test on clinician-taken samples. In the HerSwab group, the corresponding results were $71.7 \%, 46.7 \%, 61.3 \%, 58.3 \%$ for cytology; $75.0 \%, 47.7 \%, 62.9 \%$ and $61.8 \%$ for HPV test on self-taken samples and $94.3 \%, 44.4 \%, 66.7 \%$ and $87.0 \%$ for clinician-taken samples, respectively.

Conclusions. The results confirm that HPV self-sampling is not as accurate as clinician sampling when HC2 is used. All HPV tests showed a higher sensitivity in detecting CIN2+ compared to cytology. Due to non-inferior longitudinal sensitivity of HPV self-sampling compared to cytology, HPV self-sampling might be an option for non-attenders to the National Cancer Screening Programme.

Key words: HPV self-sampling; cytology; high-grade intraepithelial lesion 


\section{Introduction}

Since the introduction of the cervical cancer screening based on cell samples for cervical cytology (Pap smear) the incidence and mortality of cervical cancer has decreased dramatically., In Slovenia, the highest peak of the cervical cancer incidence was registered in 1962 when age-standardised incidence rate (world) was 27.5/100,000. Due to the opportunistic screening the incidence was decreasing till the end of the eighties and a second peak was observed in 1997. ${ }^{3}$ Organised cervical screening national program ZORA (NP ZORA) was implemented in 2003 with conventional cytology at a three-years interval in women aged 20-64. A three-year coverage of the target population with a screening test is just above $70 \%{ }^{4}$ The lowest incidence of cervical cancer in Slovenia was registered in 2017 when 85 new cases were diagnosed and the age-standardised incidence rate (world) was $4.9 / 100,000$. $^{5}$

Despite good results of NP ZORA, there are still subgroups of women who do not attend for screening. In Slovenia, the coverage of the target population with screening test is decreasing with women's age. It is below the targeted $70 \%$ in women 50 years or more. The lowest rate is in women aged 60-64 years with only $57 \%{ }^{4}$

In countries with organised screening programmes, the majority of new cases are diagnosed in women who were never screened or are underscreened. These women are often diagnosed at advanced stages. Nonattendance for screening is one of the most important risk factors for developing cervical cancer. Studies exploring screening non-attendance suggest a wide range of barriers, including fear of pain, embarrassment, shame, low perceived risk, absence of symptoms, lack of physicians, inconvenient clinic hours, forgetting an appointment, cultural barriers, low socioeconomic status, indirect costs, and worry about the result. ${ }^{6,7}$

The discovery that human papillomaviruses (HPV) are aetiologically linked with cervical cancer has led to efforts to apply this knowledge to improve cervical cancer screening. Over the last two decades, HPV testing has become a part of clinical guidelines for cervical cancer screening, triage and follow-up after treatment in several countries. ${ }^{8}$

One of the advantages of HPV testing is the possibility for women to perform self-sampling. Systematic reviews and meta-analysis published in recent years have shown that self-sampling for HPV testing may increase a population uptake of cervical cancer screening, especially when HPV self-sampling kits were mailed directly to women. ${ }^{9,10}$ HPV self-sampling is a process where a woman uses a kit to collect a vaginal sample, which is then sent for analysis by a laboratory, while sample taken by gynaecologist is obtained from the cervix. The difference between both methods has raised concerns about whether vaginal self-sampling is comparable to cervical clinician-sampling in detecting HPV. In the updated meta-analysis by Arbyn et al. in 2018, HPV assays based on polymerase chain reaction were as sensitive on self-samples as on clinical samples; however, the specificity to exclude cervical intraepithelial neoplasia grade 2 or more (CIN $2+$ ) was $2 \%$ or $4 \%$ lower on self-samples than on clinical samples. ${ }^{11} \mathrm{HPV}$ assays based on signal amplification were less sensitive on selfsamples. ${ }^{11}$

For the first time, we are presenting the results of the Slovenian HPV self-sampling pilot study in colposcopy population that was conducted one year prior the large-scale randomised trial of HPV self-sampling. ${ }^{12}$ One and four-year sensitivity, specificity, positive, and negative predictive values for CIN2+, test agreement and CIN $2+$ detection rates in women enrolled in the study were analysed. Results are stratified by the sampling modality (self, clinician), test (cytology, HPV), and self-sampling device (Qvintip, HerSwab).

\section{Participants and methods}

Women were consecutively enrolled in the study at the colposcopy clinic at the University Medical Centre Maribor and General Hospital Celje during 2014-2016 and followed by the central National Cervical Cancer Screening Registry until the end of 2019. Women with pathologic Pap test, age 20-64 years, referred for a colposcopy according to National guidelines for the management of women with pathological cervical results ${ }^{13}$, were invited to participate in prospective observational study and all included women signed informed consent. Exclusion criteria were acute kolpitis or cervicitis and pregnancy. All women performed a self-collected vaginal swab for HPV testing at the clinic prior to the gynaecological examination. After self-sampling, the gynaecologist collected two cervical smears, first for conventional cytology and second for a HPV test. After that, all women underwent colposcopy and in case of abnormal colposcopy cervical biopsy was performed. Women were managed according to the colposcopy and biopsy results and the National guidelines for the 


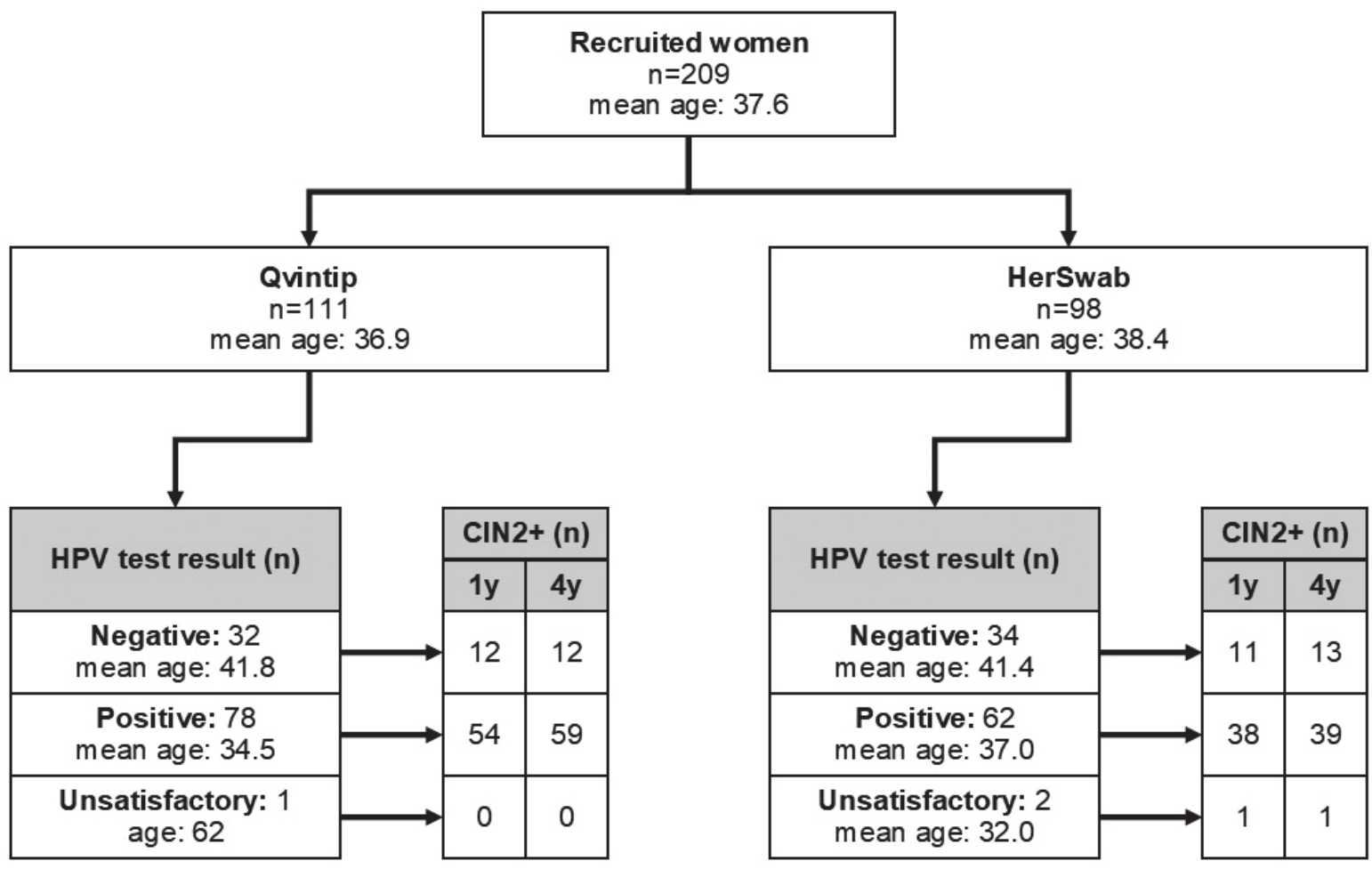

FIGURE 1. Flow chart of women enrolled in the study with results of human papillomaviruses (HPV) self-sampling and histological cervical intraepithelial neoplasia grade 2 or more $(\mathrm{CIN} 2+)$ results after one and four-year follow-up.

management of women with pathological cervical results. ${ }^{13}$ There were two self-sampling devices used in the study: Qvintip (Aprovix AB, Uppsala Sweden) and HerSwab (Eve Medical, Toronto, Canada); however, each woman used only one selfsampling device. Hybrid Capture 2 (HC2, Qiagen, Hilden, Germany) assay was used for all HPV testing. Cytological and histological evaluations were performed by certified pathologists in laboratories as part of a regular Cervical Cancer Screening Programme. In the event of having more than one histopathological result, the pathological change of the highest grade was included in the analysis. Each cytology slide was evaluated twice: at the institution performing gynaecological examination and at Institute of Oncology Ljubljana.

\section{Statistical analysis}

The Mann-Whitney U Test was used to compare whether there is a difference in age and the Chisquared test to compare proportions of positive results and CIN2+ among tester groups. Test performance for CIN 2+ was evaluated with a 4-year longitudinal sensitivity, specificity, negative predictive value (NPV), and positive predictive value (PPV). Confidence intervals (CI) for performance measures were calculated with the bootstrap method. Relative performance was calculated as a ratio of performance measures of HPV test on a self-sample vs. HPV test on a sample taken by a gynaecologist and cytology. All women in the Qvintip group had a histological follow up of at least 4 years, while two women without CIN2+ event in the HerSwab group had a histological follow up of 3 years, and 9 and 11 months, respectively. They both had negative cytology and HPV test and they were included in analysis as negative for CIN2+. All women included in the study were followed-up after 4 years according to national guidelines by personal gynaecologists who are providers of National cancer screening programme. Cumulative incidence was calculated using the Kaplan-Meier method. The duration of histological follow up was at least 5 years for all women in the Qvintip group and more than $50 \%$ of women in the HerSwab group. Women were censored at the end of histological follow up or death. One woman without CIN2+ event from the HerSwab group was censored due to death after follow up time of just below two years and two women were censored three and one month before the four years mark due to end of histological follow up. The data were obtained from the National cervical screening program registry. Agreement 


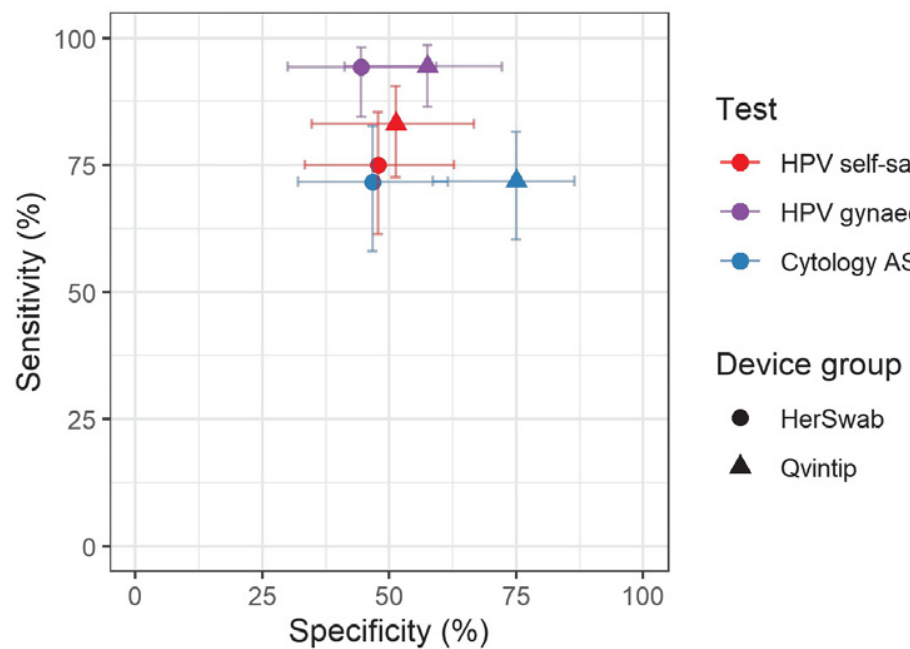

FIGURE 2. Sensitivity and specificity for cervical intraepithelial neoplasia grade 2 or more (CIN2+) by test (cytology, human papillomaviruses [HPV]), testing modality (self, clinician) and self-sampling device (Qvintip, HerSwab) after four years of followup following enrolment.

ASC-US+ = atypical squamous cells of undetermined significance or more

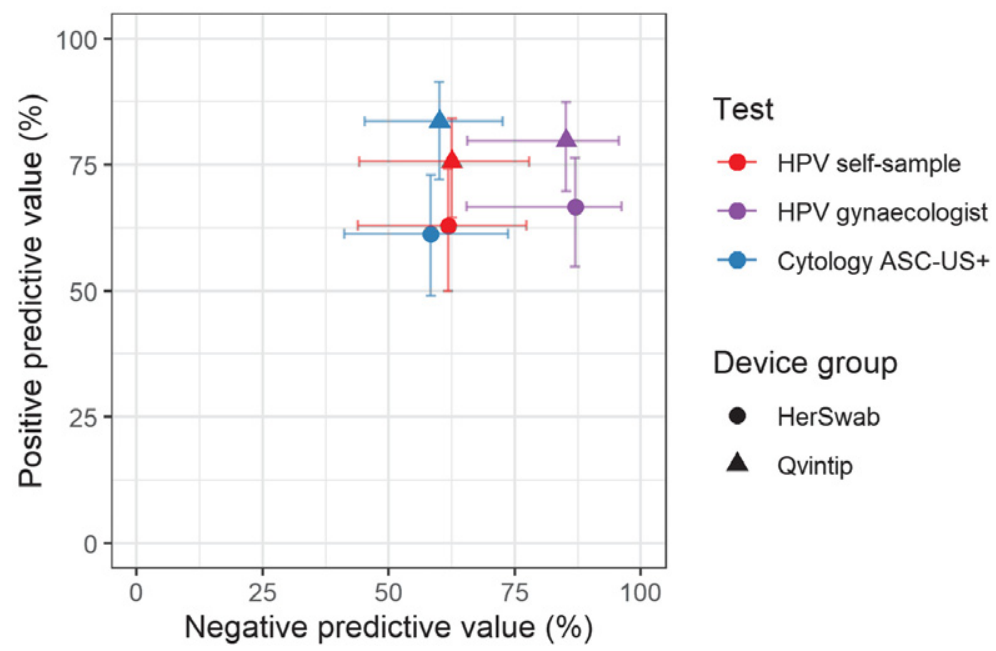

FIGURE 3. Positive predictive value and negative predictive value for cervical intraepithelial neoplasia grade 2 or more $(\mathrm{CIN} 2+)$ by test (cytology, human papillomaviruses [HPV]), testing modality (self, clinician) and self-sampling device (Qvintip, HerSwab).

ASC-US+ = atypical squamous cells of undetermined significance or more

was evaluated with overall percent agreement (OPA) and Cohen's kappa. Atypical squamous cells of undetermined significance or more (ASCUS+) was considered a positive result for cytology. Cytology results in the test performance analysis are provided by the Institute of Oncology. Women with negative colposcopy and no histology were considered negative for CIN2+. Unsatisfactory self- samples were excluded from analyses of agreement, test performance, and cumulative incidence. All analyses were conducted in R v3.6.3 ${ }^{14}$ using the significance level $\alpha=0.050$.

The study was coordinated by the Institute of Oncology Ljubljana. It was conducted in compliance with the Helsinki Declaration and was approved by the National Medical Ethics Committee at the Slovenian Ministry of Health (consents Nos. 155/03/13 and 136/04/14). All women gave their written informed consent prior to study inclusion.

\section{Results}

\section{Characteristics of the enrolled women}

A total of 209 women were enrolled in the study, of them 111 to the Qvintip and 98 to the HerSwab group (Figure 1). The mean age of the enrolled women was 37.6 years; 140 women were tested positive with a HPV self-sampling test $(67.0 \%), 66$ negative $(31.6 \%)$ and in three women, the self-taken sample was technically inadequate (1.4\%). One year after the enrolment, CIN2+ was diagnosed in 116 women $(55.5 \%)$ and four years after the enrolment in 124 (59.3\%) women.

Women in the Qvintip group were on average younger than women in the HerSwab group (mean age 36.9 vs. 38.4 years, $p=0.221$ ), had a higher probability for a positive result of HPV self-sampling $(70.3 \%$ vs. $63.3 \%, \mathrm{p}=0.283)$ and CIN2+ diagnosis after one $(59.4 \%$ vs. $51.0 \%)$ and four years of follow up $(64.0 \%$ vs. $54.1 \%, \mathrm{p}=0.146)$ (Figure 1$)$.

\section{Accuracy of HPV self-sampling}

Four-year longitudinal sensitivity and specificity of HPV self-sampling for CIN2+ were $83.1 \%$ and $51.3 \%$, respectively, in the Qvintip and $75.0 \%$ and $47.7 \%$ in the HerSwab group. Four-years longitudinal NPV and PPV were $62.5 \%$ and $75.6 \%$ in the Qvintip and $61.8 \%$ and $62.9 \%$ in the HerSwab group (Figures 2 and 3, Supplementary Table 1).

An increase in the sensitivity and NPV from cytology (blue) and HPV self-sampling (red) toward HPV test on clinician-taken samples (violet) is evident in both device groups (Figures 2 and 3, Supplementary Tables 1 and 2). The sensitivity and NPV of cytology were similar in both device groups, which was also observed for HPV test on clinician-taken samples. In HPV self-sampling, the sensitivity was higher in the Qvintip group compared to the HerSwab group (83.1 vs. 75.0); however, NPV was similar (62.5 vs. 61.8). Four- 


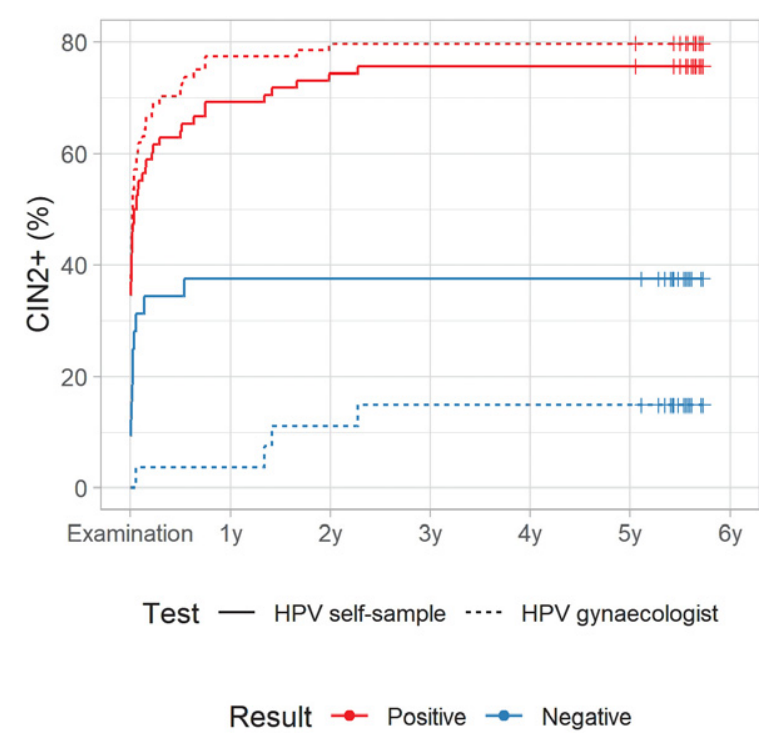

FIGURE 4A. Cumulative incidence of cervical intraepithelial neoplasia grade 2 or more (CIN2+) according to human papillomaviruses (HPV) test result in Qvintip group

year longitudinal specificity and PPV were higher in the Qvintip group compared to the HerSwab group, regardless the sampling modality and test (Figures 2 and 3, Supplementary Table 1).

Relative performance of HPV self-sampling versus cytology after four-years' follow-up in the HerSwab group with relative sensitivity (1.05), specificity (1.02), PPV (1.03), and NPV (1.01) indicate a similar accuracy of those two testing methods, which is also evident from the close position of red and blue circles in Figures 2 and 3. In the Qvintip group, the relative sensitivity of HPV selfsampling versus cytology (1.16) and relative NPV (1.04) indicates a slightly higher sensitivity with similar NPV, yet a lower specificity (relative 0.68 ) and PPV (relative 0.90 ) of self-sampling compared to cytology.

Relative sensitivity, NPV and PPV of HPV selfsampling versus HPV test of clinician-taken samples after four-years' follow-up in the HerSwab group $(0.80,0.71,0.94)$ and Qvintip group $(0.88$, 0.73 and 0.95 ) indicate lower sensitivity, NPV and PPV of self-sampling compared to HPV test on clinician samples in both device groups. However, relative specificity of HPV self-sampling compared to HPV test on clinician-taken sample indicates a similar specificity in HerSwab (1.07) and a lower one in Qvintip (0.89).

None of the differences between HPV tests on self-collected samples and cytology or HPV test on a sample taken by gynaecologists was statisti-

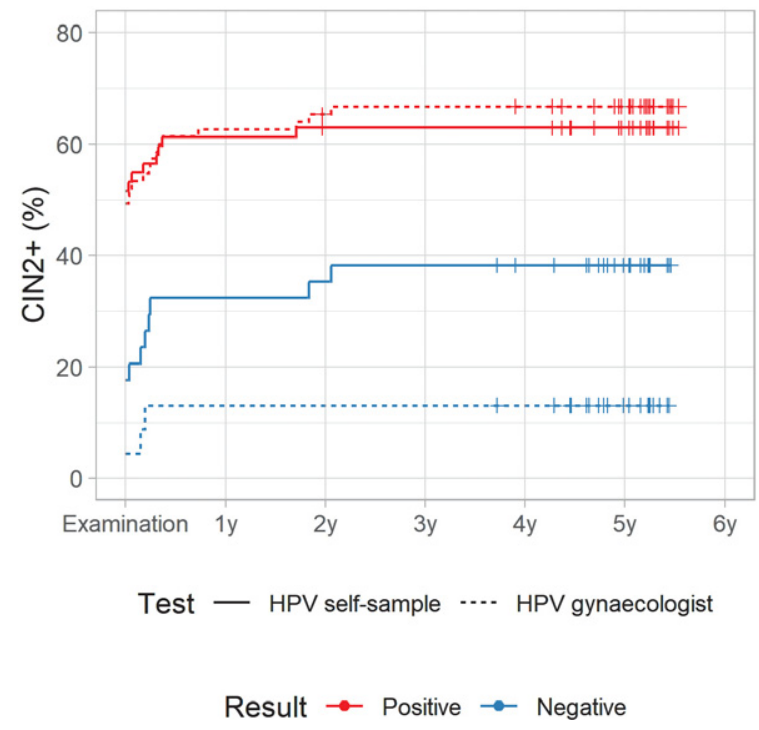

FIGURE 4B. Cumulative incidence of cervical intraepithelial neoplasia grade 2 or more (CIN2+) according to human papillomaviruses (HPV) test result in HerSwab group.

cally significant. The same applies to differences between the two self-sampling devices.

\section{Cumulative incidence of CIN2+}

Figures $4 \mathrm{~A}$ and $4 \mathrm{~B}$ show a cumulative incidence of CIN2+ in a five-year follow-up period in the Qvintip and HerSwab group according to the result of HPV self-sampling and HPV test on clinician-taken samples. Most of the CIN2+ cases were detected in the first half of a year after examination at the colposcopy clinic. There were few CIN2+ cases between year two and three and no CIN2+ in years three to five.

For HPV-negative women, the cumulative incidence of CIN2+ after four-years follow up was similar in both device groups; however, it was two-times higher after a negative HPV test on selfsamples $(37.5 \%, 95 \%$ CI: $18.3-52.2 \%$ and $38.2 \%$, 95\% CI: $19.5-52.6 \%)$ than after a negative HPV test on a clinician-taken sample $(14.8 \%, 95 \% \mathrm{CI}$ : $0.3-27.2 \%$ and $13.0 \%, 95 \%$ CI: $0.0-25.8 \%$ ). On the other hand, HPV-positive women in the Qvintip group had higher cumulative incidence of CIN2+ after four-years follow up compared to HerSwab group women. However, the difference between HPV self-sampling and a clinician-taken HPV test was small $(75.6 \%, 95 \%$ CI: $64.0-83.5 \%$ and $62.9 \%$, 95\% CI: $48.7-73.2 \%$ vs. $79.8 \%$, $95 \%$ CI: $69.1-86.8 \%$ and $66.7 \%, 95 \%$ CI: $54.1-75.9 \%$ ) (Figures $4 \mathrm{~A}$ and 4B, Suppl. Table 3). 
TABLE 1. Concordance of human papillomavirus (HPV) test results (among devices) and cytology (among laboratories)

\begin{tabular}{cccccc}
\hline & \multicolumn{2}{c}{ HPV test } & & \multicolumn{2}{c}{ Cytology } \\
\cline { 2 - 3 } \cline { 5 - 6 } & OPA $(\%)$ & kappa $(95 \% \mathrm{Cl})$ & & OPA $^{\mathrm{a}}(\%)$ & kappa $(95 \% \mathrm{Cl})$ \\
\hline Qvintip group & 81.8 & $0.534(0.349-0.718)$ & & 74.8 & $0.471(0.3029-0.641)$ \\
HerSwab group & 77.1 & $0.456(0.2569-0.655)$ & & 74.5 & \\
Total & 79.6 & $0.495(0.3599-0.632)$ & & 74.6 & $0.406(0.2059-0.607)$ \\
\hline
\end{tabular}

$\mathrm{Cl}=$ confidence interval; OPA = overall percent agreement

\section{Agreement of tests}

The overall percent agreement (OPA) between HPV test on a self-sample and a sample taken by a gynaecologist was $79.6 \%$ (kappa 0.495) and similar in both device groups (Table 1).

\section{Discussion}

This is the first time we are presenting the results of the Slovenian HPV self-sampling pilot study in the colposcopy population within the organised, population-based Slovenian cervical cancer screening programme ZORA. Four-year longitudinal accuracy and cumulative incidence of CIN2+ for HPV testing with samples taken by Qvintip and HerSwab self-sampling devices were analysed and compared to cytology and HPV tests with clinician-taken samples. The prevalence of HPV positive self-sampling results in our group of patients was $67.0 \%$. In colposocpy studies among women with abnormal cervical smears, the reported prevalence of self-collected HPV positive tests ranges between 30 and $77 \% .^{15-21}$ We found more positive HPV tests in the Qvintip group compared to the HerSwab group ( $70.3 \%$ vs $63.3 \%$ ) probably due to a lower age of women and higher prevalence of CIN2+ in the Qvintip group compared to the HerSwab group.

Our results showed a higher sensitivity to detected CIN2+ in clinician-taken samples comparing to both self-sampling devices after 1 and 4 years. Specificity was higher in clinician-taken samples in the Qvintip group (57.8\% vs. $45.5 \%$ after 1 year and $57.5 \%$ vs. $51.3 \%$ after 4 years) comparing to selfsampling; however, the specificity in the HerSwab group was slightly higher in self-sampling samples compared to clinician-taken samples $(48.9 \%$ vs. $41.7 \%$ after 1 year and $47.7 \%$ vs. $44.4 \%$ after 4 years). This finding is consistent with most of reports indicating that HPV self-sampling has a lower sensitivity compared to clinician-taken samples and with of meta-analysis results published in 2014 by Arbyn et al., which included 36 studies and more than 154,500 women. Pooled sensitivity of HPV self-sampling to detect CIN2+ was $76 \%$ and specificity $86 \%$ while the pooled sensitivity and specificity of HPV testing on self-samples was lower than HPV testing with clinician-taken samples.22

In contrary, results of a Netherlands randomised study showed no difference between self-sampling and clinician-taken sampling of CIN2+ sensitivity (self-sampling 92.9\%; clinician sampling 96.4\%) and specificity (self-sampling $93.9 \%$; clinician sampling $94.2 \%$ ) of HPV testing. ${ }^{23}$

Arbyn's meta-analysis was updated in 2017 and HPV tests were categorized into polymerase chain reaction (PCR) and signal amplification-based tests. HPV self-sampled assays based on PCR were as sensitive and specific on self-samples as on clinician samples to detect CIN2+. However, self-sampled HPV tests based on signal amplification were not as accurate for the detection of CIN2+. ${ }^{11}$

There was no statistically significant difference between the two testers used in our study regarding accordance to clinician-obtained HPV test. Other authors who compared Qvintip or HerSwab with other self-sampling devices for in their studies also did not find any differences in accordance to clinician-obtained samples. ${ }^{19,24}$

The concordance of self-performed vaginal samples and clinician-performed cervical samples has been the topic of a large number of studies. In our study, the agreement was lower as generally reported in literature, especially in the HerSwab group (OPA 77.1\%, kappa 0.456) comparing to the Qvintip group (81.8\%, kappa 0.534), although the difference between two testers was not statistically significant. In a 2005 meta-analysis by Ogilvie et al., data from 12 studies were included and kappa values between patients and clinician obtained samples ranged from $0.45-1.00 .{ }^{25} \mathrm{~A}$ systematic review and meta-analysis of 18 studies published in 2007 by Petignat et al., found a concordance between self-sampled and physician-sampled specimens 
for detection of HPV DNA in $87.0 \%$, pooled kappa value of $0.66 .{ }^{26}$ Most recent studies reported OPA between vaginal self-obtained and cervical clinician-obtained samples for the detection of HPV to be between 91.2 and $96.8 \% .^{19,27-32}$ The studies using the same self-sampling devices as in our study reported better agreement as well. In a German study, the Qvintip tester showed OPA with clinician collected sample in $89.0 \%$ (kappa 0.779), which is better than in our group of patients using the same type of self-sampling device $(81.8 \%) .{ }^{19}$ ElZein et al. reported a better agreement for HerSwab device with a kappa value of 0.84 compared to our group of patients (kappa 0.456). ${ }^{32}$

In a CASSIS study, HerSwab was used among others self-sampling devices and showed higher sensitivity (88.6\%) and specificity (58.1\%) than the one found in our study $(77.6 \%$ and $48.9 \%$ after 1 year; $75.0 \%$ and $47.7 \%$ after 4 years). ${ }^{24}$ Sensitivity $(92.4 \%)$ with clinician sampling was similar to ours and specificity $(58.7 \%)$ was similar to that in our Qvintip group. In the same study, PPVs for CIN2+ were $28.0 \%$ and $29.7 \%$ for HerSwab and clinician taken samples respectively, which is quite lower compared to results in our group using the same self-sampling device $(61.3 \%$ and $62.3 \%$ after 1 year; $62.9 \%$ and $66.7 \%$ after 4 years). ${ }^{24}$ The underlying reason is probably the different prevalence of the diseases. In our study, CIN2+ was diagnosed in 116 women $(55.5 \%)$ one year after enrolment and four years after enrolment in 124 (59.3\%) women. In a CASSIS study of 1217 women, 1076 had complete results for HPV and cytology; $148(13.8 \%)$ had CIN1, 147 (13.7\%) had CIN2/3, and $5(0.5 \%)$ had cancer.

Relative sensitivity seems somehow higher in Qvintip compared to HerSwab, HPV self-sampling versus HPV test on clinician-taken samples $(83.1 / 94.4=0.88$ vs. $75.0 / 94.3=0.80)$ as well as in HPV self-sampling versus cytology (83.1/71.8 = 1.16 vs. $75 \cdot 0 / 71.7=1.05)$. However, relative specificity seems slightly higher in HerSwab compared to Qvintip, HPV self-sampling versus HPV test on clinician-taken samples $(51.3 / 57.5=0.89$ vs. $47.7 / 44.4=$ $1.07)$, as well as in HPV self-sampling versus cytology $(51.3 / 75.0=0.68$ vs. $47.7 / 46.7=1.02)$.

The sensitivity of cytology and HPV test on clinician-taken samples in our colposcopy study were similar between the Qvintip (71.8\% and 94.4\%) and HerSwab group (71.7\% and $94.3 \%$ ) and higher than in the recent Cochrane database systematic review of comparisons of $\mathrm{HC} 2$ results versus conventional cytology (ASC-US+ threshold) in the general population, where the pooled sensitivity of cytology was
$62.5 \%$ and for HPV test on clinician-taken samples $89.9 \% .^{33}$

In a CASSIS study cytology ASC-US+ was $80.2 \%$ sensitive and $61.4 \%$ specific for CIN2+. ${ }^{24}$ The systematic review and meta-analysis in low and middle-income countries included more than 700 cervical cancers from 23 studies and found pooled sensitivity of cytology for cancer $79.4 \%$ at a cut-off HSIL+. ${ }^{34}$ On the other hand, Greek study authors reported about a very low sensitivity of cytology, $13.6 \%$ at cut-off HSIL. ${ }^{35}$

It is known that cervical cytology has limited sensitivity and that results may vary between different pathologists and laboratories and that HPV testing detects more cervical intraepithelial neoplasia than cytology. Therefore, some countries (Netherlands, Great Britain) implemented HPV testing as a primary screening test. ${ }^{36,37}$ Randomised clinical trials were conducted in many developed countries to evaluate primary HPV testing for cervical cancer screening in an organized program setting.

However, HPV testing has limitations. One of them is natural history of HPV infection, which is very common in young, sexually active women, but in majority of cases the infection is transient. Therefore, is not reasonable to use HPV testing for cervical screening in young women. Also, in elderly women positive HPV test does not necessarily imply the presence of precancerous cervical lesion. On the other hand, women with negative HPV test, have an extremely low risk of developing cervical cancer.

The European Guidelines do not recommend primary HPV screening before the age of 30 and are in favour of screening starting at the age of 35 . Since HPV testing on self-taken samples is less accurate than on clinician-taken samples, self-sampling is recommended only for non-attenders in local settings. ${ }^{38}$ As HPV testing has a higher sensitivity than cytology in elderly population ${ }^{39-41}$, selfsampling and HPV testing may be a good alternative for non-attenders to cervical screening in this age group.

\section{Conclusions}

Self-sampling for HPV testing was less accurate compared to HPV testing on clinician-taken samples; however, there was no statistically significant difference between two testers used in our study. Conventional cytology was found to have a lower sensitivity for CIN2+ than HPV testing. Our study 
included relatively young women with an average age below 40 and we assume that the difference in sensitivity may be even greater in elderly population. The self-sampled HPV test is no less sensitive than cytology and can be safely applied in non-responders to the national ZORA program. However, this kind of screening might also be more suitable for women who used to attend regular gynaecological check-ups.

\section{Acknowledgment}

The authors would like to thank Maja Primic Žakelj, Mojca Florjančič and Mojca Kuster from the NP ZORA coordination centre at the Institute of Oncology Ljubljana for their leading role in the coordination of the study. Special thanks goes to the gynaecologists, nurses and other experts who were involved in the study at all three institutions: Alenka Repše Fokter, Mateja Marčec, Maja Pakiž, Tatjana Kodrič, Andrej Cokan, Sarah Dobnik, Jure Knez, Marica Miklavc, Marcela Živko, Aleksandra Muhič, Uršula Salobir Gajšek, Jakob Koren, Veronika Kloboves Prevodnik and Nataša Nolde. Last but not least, we also thank all the women who participated in the study.

Study was approved for public funding by the Slovenian Research Agency (ARRS) (project number L3-5512). It was financed by ARRS and Ministry of Health of Republic of Slovenia. Materials used in the study were obtained free of charges or with discount from manufacturers. ARRS, Ministry of Health and manufacturers didn 't have any role in the design of the study, study execution, analyses, interpretation of the data, or decision to submit results.

\section{References}

1. Meggiolaro A, Unim B, Semyonov L, Miccoli S, Maffongelli E, La Torre G. The role of Pap test screening against cervical cancer: a systematic review and meta-analysis. Clin Ter 2016; 167: 124-39. doi: 10.7417/CT.2016.1942

2. Jansen EEL, Zielonke N, Gini A, Anttila A, Segnan N, Vokó Z, et al. Effect of organised cervical cancer screening on cervical cancer mortality in Europe: a systematic review. Eur J Cancer 2020; 127: 207-23. doi: 10.1016/j. ejca.2019.12.013

3. Zadnik V, Primic Zakelj M, Lokar K, Jarm K, Ivanus U, Zagar T. Cancer burden in Slovenia with the time trends analysis. Radiol Oncol 2017; 51: 47-55. doi: 10.1515/raon-2017-0008

4. National Cervical Cancer Screening Programme ZORA. DP ZORA: kazalniki. [internet]. [cited 2020 Jan 6]. Available at: https://zora.onko-i.si/publikacije/ kazalniki/

5. National Cervical Cancer Screening Programme ZORA. [internet]. [cited 2020 Jan 6]. Available at: https://zora.onko-i.si/
6. Benett KF, Waller J, Chorley AJ, Ferrer RA, Haddrell JB, Marlow LAV. Barriers to cervical screening and interest in self-sampling among women who actively decline screening. Med Screen 2018; 25: 211-7. doi: $10.1177 / 0969141318767471$

7. Logan L, Mcilfatrick S. Exploring women's knowledge, experiences and perceptions of cervical cancer screening in an area of social deprivation. Eur J Cancer Care 2011; 20: 720-7. doi: 10.1111/j.1365-2354.2011.01254.x

8. Arbyn M, Ronco G, Anttila A, Meijer CJLM, Poljak M, Ogilvie G, et al. Evidence regarding human papillomavirus testing in secondary prevention of cervical cancer. Vaccine 2012; 30: 88-99. doi: 10.1016/j.vaccine.2012.06.095

9. Verdoodt F, Jentschke M, Hillemanns P, Racey CS, Snijders PJ, Arbyn M. Reaching women who do not participate in the regular cervical cancer screening programme by offering self-sampling kits: a systematic review and meta-analysis of randomised trials. Eur J Cancer 2015; 51: 2375-85. doi: 10.1016/j.ejca.2015.07.006

10. Yeh PT, Kennedy CE, de Vuyst $H$, Narasimhan M. Self-sampling for human papillomavirus (HPV) testing: a systematic review and meta-analysis. BM Global Health 2019; 4: e001351. doi: 10.1136/bmjgh-2018-001351

11. Arbyn M, Smith SB, Temin S, Sultana F, Castle P; Collaboration on SelfSampling and HPV Testing. Detecting cervical precancer and reaching underscreened women by using HPV testing on self samples: updated meta-analyses. BMJ 2018; 363: k4823. doi: 10.1136/bmj.k4823

12. Ivanus U, Jerman T, Fokter Repse A, Takac I, Prevodnik VK, Marcec M, et al. Randomised trial of HPV self-sampling among non-attenders in the Slovenian cervical screening programme ZORA: comparing three different screening approaches. Radiol Oncol 2018; 52: 399-412. doi: 10.2478/ raon-2018-0036

13. Guidelines for management of women with cervical precancerous lesions. Ursic-Vrscaj M, Rakar S, Možina A, Kobal B, Takač I, Deisinger I, editors. Ljubljana; Institute of Oncology Ljubljana; 2011. [internet]. [cited 2020 Jan 20]. Available at: https://zora.onko-i.si/fileadmin/user_upload/dokumenti/ strokovna_priporocila/2011_Smernice_web.pdf

14. R Core Team. R: a language and environment for statistical computing. Vienna: R Foundation for Statistical Computing; 2020. [internet]. [cited 2020 Jan 20]. Available at: http://www.R-project.org/

15. Jentschke $M$, Soergel $P$, Hillemanns P. Evaluation of multiplex real time PCR assay for the detection of human papillomavirus infection on self-colleceted cervicovaginal lavage samples. J Clin Virol 2013; 193: 131-4. doi: 10.1016/j. jviromet.2013.05.009

16. Aiko KY, Yoko M, Saito OM, Ryoko A, Yasuyo M, Mikiko AS, et al. Accuracy of self-collected human papillomavirus samples from Japanese women with abnormal cervical cytology. J Obstet Gynaecol Res 2017; 43: 710-7. doi: org/10.1111/jog.13258

17. Nobbenhuis MAE, Helmerhorst TJM, Van den Brule AJC, Rozendaal L, Jaspar $\mathrm{LH}$, Voorhorst FJ, et al. Primary screening for high risk HPV by home obtained cervicovaginal lavage is an alternative screening tool for unscreened women. J Clin Pathol 2002; 55: 435-9. doi: 10.1136/jcp.55.6.435

18. Sellors JW, Lorincz AT, Mahony JB, Mielzynska I, Lytwyn A, Roth P, et al. Comparison of self-collected vaginal. Vulvar and urine samples with physician-collected cervical samples for human papillomavirus testing to detect high-grade squamous intraepithelial lesions. CMAJ 2000: 163: 513-8. PMID: 11006761

19. Jentschke M, Chen K, Arbyn M, Hertel B, Noskowicz M, Soergel P, et al Direct comparison of two vaginal self-sampling devices for the detection of human papillomavirus infections. J Clin Virol 2016; 82: 46-50. doi: 10.1016/j. jcv.2016.06.016

20. Darlin L, Bergfeldt C. Forslund O, Hénic E, Dillner J, Kannisto P. Vaginal selfsampling without preservative for human papillomavirus testing shows good sensitivity. J Clin Virol 2013; 56: 52-6. doi: 10.1016/j.jcv.2012.09.002

21. Seo SS, Song YS, Kim JW, Park NH, Kang SB, Lee HP. Good correlation oh HPV DNA test between self-collected vaginal and clinician-collected cervical samples by the oligonucleotide microarray. Gynecol Oncol 2006; 102: 67-73. doi: 10.1016/j.ygyno.2005.11.030

22. Arbyn M, Verdoodt F, Snijeders PJF, Verhoef VMJ, Suonio E, Dillner L, et al. Accuracy of human papillomavirus testing on self-collected versus cliniciancollected samples: a meta-analysis. Lancet Oncol 2014; 15: 172-83. doi: 10.1016/S1470-2045(13)70570-9 
23. Polman NJ, Ebisch RMF, Heideman DAM, Melchers WJG, Bekkers DAM, Molijn AC, et al. Performance of human papillomavirus testing on selfcollected versus clinician-collected samples for the detection of cervical intraepithelial neoplasia of grade 2 or worse: a randomised, paired screenpositive, non-inferiority trial. Lancet Oncol 2019; 20: 229-38. doi: 10.1016/ S1470-2045(18)30763-0

24. El-Zein $M$, Bouten $S$, Louvanto $K$, Gilbert $L$, Gotlieb $W$, Hemnings $R$, et al. Validation of a new HPV self-sampling device for cervical cancer screening: the cervical and self-sample in screening (CASSIS) study. Gynecol Oncol 2018; 149: 491-7. doi: 10.1016/j.ygyno.2018.04.004

25. Ogilvie GS, Patrick DM, Schulzer M, Sellors JW, Petric M, Chambers K, et al. Diagnostic accuracy of self-collected vaginal specimens for human papillomavirus compared to clinician collected human papillomavirus specimens: a meta-analysis. Sex Transm Infect 2005; 8: 207-12. doi: 10.1136/ sti.2004.011858

26. Petignat $P$, Faltin DL, Bruchim I, Tramèr MR, Franco EL, Coutlée F. Are selfcollected samples comparable to physician-collected cervical specimens for human papillomavirus DNA testing? A systematic review and meta-analysis. Gynecol Oncol 2007; 105: 530-5. doi: 10.1016/j.ygyno.2007.01.023

27. Boggan JC, Walmer DK, Henderson G, Chakhtoura N, McCarthy SH, Beauvais $\mathrm{HJ}$, et al. Vaginal self-sampling for human papillomavirus infection as a primary cervical cancer screening tool in a Haitian population. Sex Transm Dis 2015; 42: 655-9. doi: 10.1097/OLQ.0000000000000345

28. Leinonen MK, Schee K, Jonassen CM, Lie AK, Nystrand CF, Rangberg A, et al. Safety and acceptability of human papillomavirus testing of self-collected specimens: a methodologic study of the impact of collection devices and HPV assays on sensitivity for cervical cancer and high-grade lesions. J Clin Virol 2017; 9: 22-30. doi: 10.1016/j.jcv.2017.12.008

29. Toliman P, Badman SG, Gabuzzi J Silim S, Forereme L, Kumbia A et al. Field evaluation of Xpert HPV point-of-care test for detection of human papillomavirus infection by use of self-collected vaginal and clinician-collected cervical specimens. J Clin Microbiol 2016; 54: 1734-7. doi: 10.1128/JCM.0052916

30. Obiri-Yeboah D, Adu-Sarkodie Y, Djigma F, Hayfron-Benjamin A, Abdul L, Simpore J, et al. Self-collected vaginal sampling for the detection of genital human papillomavirus (HPV) using careHPV among Ghanaian women. BMC Womens Health 2017; 17: 86. doi: 10.1186/s12905-017-0448-1

31. Ketelaars PJW, Bosgraaf RP, Siebers AG, Massuger LFAG, van der Linden JC, Wauters CAP, et al. High-risk human papillomavirus detection in selfsampling compared to physician-taken smear in a responder population of the Dutch cervical screening: results of the VERA study. Prev Med 2017; 101: 96-101. doi: 10.1016/j.ypmed.2017.05.021

32. El-Zein M, Bouten S, Louvanto K, Gilbert L, Gotlieb WH, Hemmings R, et al. Predictive value of HPV testing in self-collected and clinician-collected samples compared with cytology in detecting high-grade cervical lesions. Cancer Epidemiol Biomark Prev 2019; 28: 1134-40. doi: 10.1158/1055-9965.EPI-181338

33. Koliopoulos G, Nyaga VN, Santesso N, Bryant A, Martin-Hirsch PP, Mustafa RA, et al. Cytology versus HPV testing for cervical cancer screening in the general population. Cochrane Database Syst Rev 2017; 8: CD008587. doi: 10.1002/14651858.CD008587.pub2

34. Castanov A, Landy R, Michalopoulos D, Bhudia R, Leaver H, Qiao YL, et al. Systematic review and meta-analysis of individual patient data to assess the sensitivity of cervical cytology for diagnosis of cervical cancer in lowand middle-income countries. J Glob Oncol 2017; 3: 524-38. doi: 10.1200/ JGO.2016.008011

35. Pesic A, Krings A, Hempel M, Preyer R, Chatzistamatius K, Agorastos T, et al. CIN2+ detection of the HPV DNA Array genotyping assay in comparison with the Cobas 4800 HPV test and cytology. Virol J 2019; 16: 92. doi: 10.1186/ s12985-019-1197-6

36. Polman NJ, Snijders PJF, Kenter GG, Berkhof J, Meijer CJLM. HPV-based cervical screening: rationale, expectations and future perspectives of the new Dutch screening programme. Prev Med 2019; 119: 108-17. doi: /10.1016/j. ypmed.2018.12.021

37. Rebolj M, Rimmer J, Denton K, Tidy J, Mathev C, Ellis K, et al. Primary cervical screening with high risk human papillomavirus testing: observational study. BMJ 2019; 364: 240. doi: https://doi.org/10.1136/bmj.1240
38. von Karsa L, Arbyn M, De Vuyst H, Dillner J, Dillner L, Franchesci S, et al. European guidelines for quality assurance in cervical cancer screening. Summary of the supplements on HPV screening and vaccination. Papillomavirus Res 2015; 1: 22-31. doi: /10.1016/j.pvr.2015.06.006

39. Ronco G, Giorgi-Rossi P, Carozzi F, Confortini M, Dalla Palma P, Del Mistro A, et al. New Technologies for Cervical Cancer screening (NTCC) Working Group. Efficacy of human papillomavirus testing for the detection of invasive cervical cancers and cervical intraepithelial neoplasia: a randomised controlled trial. Lancet Oncol 2010; 11: 249-57. doi: 10.1016/S14702045(09)70360-2

40. Hermansson RS, Olovsson M, Hoxell E, Lindström AK. HPV prevalence and HPV-related dysplasia in elderly women. PLoS One 2018; 13: e0189300. doi: 10.1371/journal.pone.0189300

41. Lindström AK, Hermansson RS, Gustavsson I, Hedlund Lindberg J, Gyllensten $\mathrm{U}$, Olovsson M. Cervical dysplasia in elderly women performing repeated self-sampling for HPV testing. PLoS One 2018; 13: e0207714. doi: 10.1371/ journal.pone.0207714 